\title{
Queimaduras: características dos casos tratados em um hospital escola em Ribeirão Preto (SP), Brasil
}

\author{
Lídia Aparecida Rossi, ${ }^{1}$ Rita de Cássia de P. Barruffini, ${ }^{2}$ \\ Telma R. Garcia ${ }^{3}$ e Tânia C. M. Chianca ${ }^{4}$
}

RESUMO As queimaduras são um problema de saúde significativo no Brasil. Contudo, até hoje, existem poucos dados disponíveis para orientar programas de tratamento e prevenção. Com o intuito de preencher esta lacuna, o presente estudo examina os tipos de acidentes e as características dos 138 pacientes que sofreram queimaduras e foram internados na unidade de queimados do Hospital Escola da Universidade de São Paulo em Ribeirão Preto, estado de São Paulo, Brasil, entre novembro de 1991 e dezembro de 1993. A partir do prontuário dos pacientes, foram coletados dados sobre idade, sexo, área do corpo afetada pela queimadura, profundidade e extensão da queimadura, agente causador e circunstância em que ocorreram as queimaduras. Do total de casos, $71 \%$ ocorreram na casa da vítima e $50 \%$ envolveram crianças. A água fervente foi responsável por 59\% dos acidentes entre as crianças com menos de 3 anos; o álcool utilizado em casa foi responsável por $40 \%$ dos acidentes com crianças entre 7 e 11 anos, o grupo mais atingido entre os pacientes pediátricos. Entre os adultos, os pacientes com idade ente 20 e 39 anos foram os mais atingidos (23\%); destes, 84\% eram do sexo masculino. Dezessete por cento do número total de casos envolveu adultos do sexo masculino em situações de trabalho. Os pacientes adultos do sexo feminino queimaram-se em situações domésticas (67\%) e tentativas de suicídio (33\%). Setenta e cinco por cento dos pacientes que tentaram o suicídio através da queimadura eram do sexo feminino. As estratégias para redução da incidência do trauma térmico deveriam atender a dois pontos principais: a eliminação de fatores de risco no próprio ambiente e a implementação de programas educativos, veiculados principalmente através de meios de comunicação como o rádio e a televisão.

A queimadura está entre os traumas mais graves, pois, além dos problemas físicos que podem levar o paciente à morte, pode acarretar outros proble- mas de ordem psicológica e social (1-3). No Brasil, os dados estatísticos sobre as lesões por queimaduras são escassos. Contudo, estes dados são im-

\footnotetext{
Universidade de São Paulo, Escola de Enfermagem de Ribeirão Preto, Departamento de Enfermagem Geral e Especializada, Ribeirão Preto, SP, Brasil. Correspondência e pedidos de separatas devem ser enviados a esta autora no seguinte endereço: Avenida Bandeirantes 3900, Campus de Ribeirão Preto, CEP 14040-902, Ribeirão Preto, SP, Brasil. Telefone: +55-16-633-3271; fax: +55-16-6023416. Email: rizzardo@glete.eerp.usp.br
}

\footnotetext{
2 Universidade de São Paulo, Hospital das Clínicas da Faculdade de Medicina de Ribeirão Preto, Seção de Enfermagem de Queimados, Ribeirão Preto, SP, Brasil.

3 Universidade Federal da Paraíba, Departamento de Saúde Pública, João Pessoa, PA, Brasil.

4 Universidade Federal de Minas Gerais, Escola de Enfermagem, Departamento de Enfermagem Fundamental, Belo Horizonte, MG, Brasil.
}

portantes para que se possa compreender a magnitude do problema e para que se possa identificar as populações mais atingidas e as circunstâncias nas quais as queimaduras ocorrem, de forma que seja possível implementar programas de prevenção. O levantamento de dados epidemiológicos também é importante para a organização de unidades especializadas no tratamento de pacientes portadores de queimaduras; existem, no Brasil, poucos centros especializados no atendimento 
de queimados, de forma que muitas das vítimas de queimaduras são internadas em hospitais que não estão equipados para atendê-las.

Em nossa experiência profissional em uma unidade de queimados na cidade de Ribeirão Preto, estado de São Paulo, observamos um alto número de acidentes por queimaduras com crianças e adultos em idade produtiva. Observamos, também, que muitos desses acidentes poderiam ser evitados através da implementação de programas educativos. Nesse sentido, o objetivo do presente estudo foi descrever os agentes causadores de queimaduras e as circunstâncias em que as queimaduras ocorrem, assim como estabelecer a distribuição por sexo e idade dos pacientes que sofreram trauma térmico e foram tratados na unidade de queimados do Hospital das Clínicas da Faculdade de Medicina de Ribeirão Preto, Universidade de São Paulo.

Os dados foram coletados através de levantamento dos registros em prontuário de pacientes internados na unidade no período de novembro de 1991 a dezembro de 1993. O estudo foi aprovado pelo Comitê de Ética em Pesquisa do Hospital das Clínicas. A unidade de queimados possui dez leitos e nela são atendidos adultos e crianças oriundos, principalmente, da região do interior do estado de São Paulo e, eventualmente, de outros estados. A média de permanência dos pacientes gira em torno de 45 dias.

Coletou-se informações sobre idade, sexo, área corporal atingida pela queimadura, profundidade, extensão da queimadura, agente causador e circunstância ou tipo de acidente (por exemplo, acidentes de trabalho, acidentes de automóvel, etc.). Foram consultados os prontuários dos 138 pacientes registrados no livro de internação e alta da unidade. Todos esses pacientes foram incluídos no estudo.

Os dados referentes a agente causador das queimaduras foram agrupados da seguinte forma: agente químico (queimaduras causadas por álcali ou ácidos); agente térmico (queimaduras causadas por agentes inflamáveis, líquidos quentes, metais quentes, brasas e chama direta); e agente elétrico (queimaduras causadas por corrente elétrica).

Os dados referentes a tipo de acidente foram agrupados nas seguintes categorias: acidente de trabalho, quando as queimaduras tinham relação com o trabalho da vítima; acidente automobilístico, quando as queimaduras eram resultantes de acidentes de trânsito envolvendo automóveis ou outros veículos; acidente doméstico, quando as queimaduras ocorreram nas dependências ou proximidades do lar; agressão, quando as queimaduras foram causadas por agressão por parte de outras pessoas; e tentativa de suicídio.

As figuras 1 e 2 apresentam a síntese dos principais resultados deste estudo. Do total de 138 pacientes atendidos pela unidade no período do estudo, $69(50 \%)$ tinham entre 0 e 11 anos. Dentre todos os pacientes pediátricos, os meninos entre 7 e 11 anos foram os mais atingidos (67\%). Todos estes pacientes foram vítimas de queimaduras por acidentes domésticos, causadas por agente térmico. Constatamos que, entre as crianças de 0 a 11 anos, a água fervente foi o agente responsável por 33\% dos acidentes, atingindo principalmente as crianças menores de 3 anos. O álcool foi responsável por $40 \%$ dos acidentes na faixa dos 7 aos 11 anos (figura 1).
A chama direta foi responsável por $16 \%$ das queimaduras entre todas as crianças menores de 11 anos. Outros agentes térmicos envolvidos em acidentes com crianças nesta faixa etária foram metal quente $(1,5 \%)$, óleo diesel $(1,5 \%)$ e brasa $(6 \%)$.

As crianças menores de 11 anos apresentaram queimaduras de segundo grau em 39\% dos casos; de segundo e terceiro graus, em diferentes áreas do corpo, em $40 \%$ dos casos; e de terceiro grau em $8 \%$ dos casos. Não encontramos informações sobre a profundidade da queimadura em $13 \%$ dos prontuários nessa faixa etária. As regiões do tórax anterior e membros superiores foram as mais atingidas nas crianças até 11 anos de idade, seguidas pela cabeça, pescoço, tórax posterior e coxas. Quanto à extensão das queimaduras, constatamos que $62,5 \%$ das crianças apresentaram queimaduras que atingiram até $20 \%$ da superfície corporal; 34\% apresentaram queimaduras em 20 a $40 \%$ da superfície corporal; e 3\% tiveram de 40 a $60 \%$ da superfície corporal atingidas. Os pacientes com idade entre 12 e 19 anos, que totalizaram $10 \%$ dos casos, foram incluídos no grupo de pacientes adultos.

Depois do grupo dos 7 aos 11 anos, os pacientes com idade entre 20 e 39 anos foram os mais atingidos pelo

FIGURA 1. Distribuição dos pacientes menores de 11 anos atendidos na unidade de queimados do Hospital de Clínicas da Faculdade de Medicina de Ribeirão Preto da Universidade de São Paulo, Brasil, conforme agente térmico causador da queimadura e faixa etária. Os dados foram coletados entre 1991 e 1993

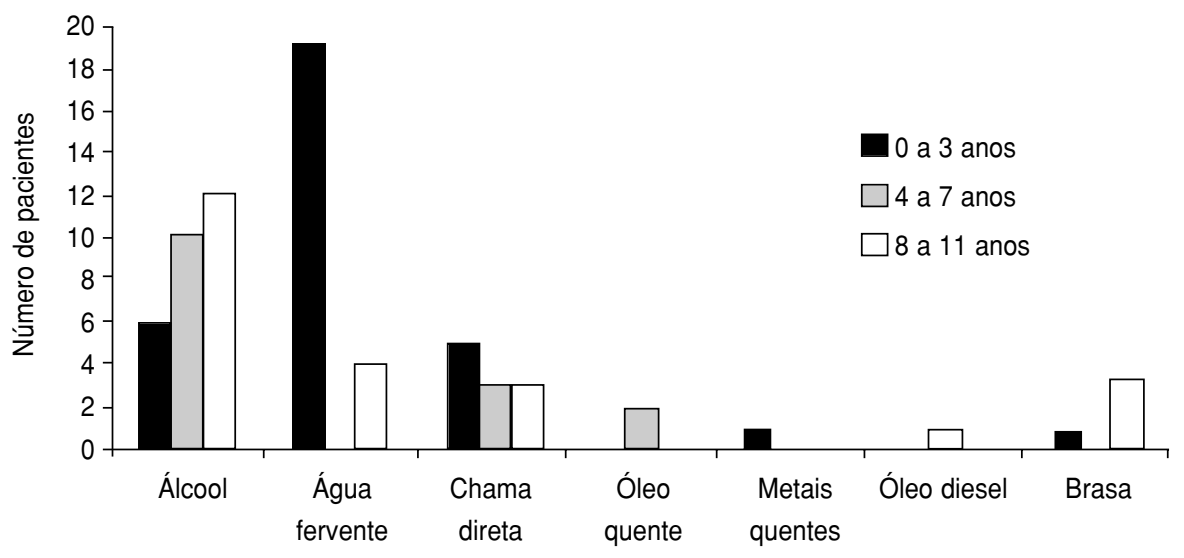


FIGURA 2. Distribuição dos pacientes com idade superior a 11 anos, de ambos os sexos, atendidos na Unidade de Queimados do Hospital de Clínicas da Faculdade de Medicina de Ribeirão Preto da Universidade de São Paulo, Brasil, conforme tipo de acidente e agente causador da queimadura. Os dados foram coletados entre 1991 e 1993

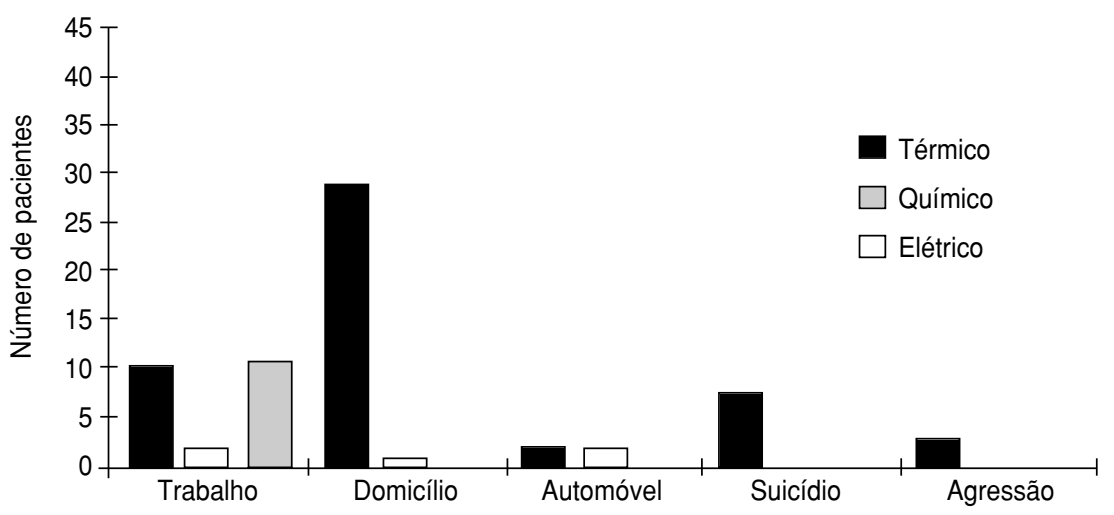

trauma térmico (23\%); destes, $84 \%$ eram do sexo masculino. Os acidentes de trabalho aparecem como principal tipo de acidente $(17 \%)$ depois dos acidentes domésticos (71\%), tendo atingido $55 \%$ dos pacientes entre 20 e 39 anos. Os acidentes de trabalho, que atingiram 55\% dos pacientes com idade entre 20 e 39 anos, foram causados por eletricidade (48\%), agentes térmicos $(43,5 \%)$ e agentes químicos (9\%) (figura 2). Entre os agentes térmicos, os principais causadores de queimaduras foram o álcool e as chamas em situações de incêndio. As queimaduras elétricas afetaram unicamente os pacientes homens em situações caracterizadas como acidentes de trabalho. As lesões por queimaduras decorrentes de agressão e acidentes automobilísticos atingiram unicamente os pacientes do sexo masculino com mais de 11 anos.

A maior parte dos pacientes vítimas de acidentes de trabalho apresentaram queimaduras de segundo e terceiro graus, sendo que $52 \%$ foram atingidos em 20 a $40 \%$ da superfície corporal. As áreas atingidas foram principalmente o tórax anterior e posterior e os membros superiores.

Os pacientes adultos do sexo feminino queimaram-se em situações domésticas (67\%) e tentativas de suicídio (33\%). Os acidentes domésticos com pacientes adultos do sexo feminino ocorreram principalmente na cozinha, envolvendo a utilização de fogões com panelas mal adaptadas e a realização de frituras. Considerando o total de pacientes que tentaram o suicídio através da queimadura, $75 \%$ eram do sexo feminino. Detectamos que todas as tentativas de suicídio foram realizadas com a utilização do álcool. As áreas corporais mais atingidas, entre as mulheres, foram cabeça, pescoço, tórax anterior e posterior e membros superiores. As queimaduras nessas regiões geralmente ocorrem quando o agente causador é atirado sobre a pessoa.

Os resultados do presente estudo indicam que a maior parte dos acidentes por queimaduras está relacionada com atividades realizadas no ambiente doméstico, atingindo, principalmente, as crianças.

O álcool foi o agente causador de grande parte dos acidentes. Este produto é utilizado em nosso país no ambiente doméstico para limpeza e também como substância inflamável e, muitas vezes, permanece ao alcance de crianças. As queimaduras provocadas por álcool são mais profundas que as provocadas por água fervente, pois o tempo de exposição da pele ao calor em queimaduras causadas por líquido inflamável é maior.

Moradias precárias, sem energia elétrica, que congregam materiais in- flamáveis para utilização em lamparinas e fogões improvisados, facilitam a ocorrência de incêndios, provocando queimaduras por chamas diretas e atingindo principalmente crianças menores de 3 anos, que, muitas vezes, estão dormindo no momento do acidente.

As áreas atingidas pela queimadura, em crianças menores de 3 anos, são, principalmente, a cabeça, tórax anterior e posterior. Isto ocorre porque os líquidos quentes, na maioria das vezes, são despejados sobre as crianças em acidentes que ocorrem nas proximidades do fogão. Os acidentes com líquidos inflamáveis geralmente ocorrem quando as crianças estão brincando com esses produtos, atingindo principalmente o tórax anterior e os braços.

As crianças do sexo masculino e com idade entre 7 e 11 anos foram as mais atingidas por queimaduras. Nesta faixa etária, as queimaduras parecem estar relacionados com as brincadeiras com álcool e outros materiais inflamáveis que, em nossa cultura, são mais comuns entre os meninos. Um estudo realizado na França (4) também apontou as crianças do sexo masculino como as mais atingidas pelo trauma térmico.

Os pacientes adultos do sexo masculino sofreram queimaduras em situações que envolveram, principalmente, o trabalho. Essas pessoas, com idades entre 20 e 39 anos, representam um grupo que se encontra no período mais produtivo da vida. Países como Irlanda (5), Austrália (3) e Índia (6), embora com características econômicas e culturais distintas, apresentaram resultados semelhantes.

Os acidentes de trabalho cujas queimaduras são provocadas por chamas ocorrem, muitas vezes, em situações de queimadas da cana de açúcar, ou seja, incêndios provocados nos canaviais durante a colheita da cana, muito comuns na nossa região. As queimaduras decorrentes de acidentes de trabalho podem levar o paciente a apresentar um quadro de desesperança, em função da súbita interrupção do processo de trabalho e das seqüelas da queimadura (7).

A maior parte dos acidentes que ocorreram no ambiente doméstico ou 
de trabalho poderiam ter sido evitados se medidas preventivas fossem implementadas. As estratégias para redução da incidência do trauma térmico deveriam atender a dois pontos principais: a eliminação de fatores de risco no próprio ambiente, envol- vendo a modificação de produtos, a legislação que normatiza a construção de edifícios, o controle de áreas para fumantes, a instalação de detectores de fumaça; e a implementação de programas educativos, veiculados principalmente através de meios de comuni- cação como o rádio e a televisão (3). Além disso, é importante que outros estudos se dediquem a coletar dados sobre a ocorrência de queimaduras em outras regiões e com grupos etários específicos.

\section{REFERÊNCIAS}

1. Artz CP, Moncrief JA, Pruitt BA. Queimaduras. Rio de Janeiro: Interamericana; 1980.

2. Monahan F, Drake T, Neighbors M. Nursing care of adults. Philadelphia: Saunders Co; 1994.

3. Duggan D, Quine S. Burn injuries and characteristics of burn patients in New South Wales, Australia. Burns 1995;21(2):83-89.

4. Mercier C, Blond MH. Epidemiological survey of childhood burn injuries in France. Burns 1996;22(1):29-34.
5. Carroll SM, Gough M, Eadie PA, McHugh M, Edwards G, Lawlor D. A 3-year epidemiological review of burn unit admissions in Dublin, Ireland: 1988-91. Burns 1995;21(5): 379-382.

6. Subrahmanyam M. Epidemiology of burns in a district hospital in Western India. Burns 1996;22(6):439-442.

7. Batista MC. O acidentado do trabalho por queimadura na região de Ribeirão Preto [Dis- sertação de mestrado]. São Paulo: Pontifícia Universidade Católica de São Paulo; 1992.

Manuscrito recebido em 10 de março de 1997. Aceito em versão revisada em 5 de agosto de 1998 .

ABSTRACT Burns are a significant health problem in Brazil, but there have been few data collected that could shape treatment and prevention programs. To help overcome that knowledge gap, this study looked at the types of accidents and the characteristics of

Burns: Characteristics of patients treated in a teaching hospital in Ribeirão Preto, Brazil 138 burn patients admitted into the burn unit of the University of São Paulo Hospital in Ribeirão Preto, state of São Paulo, Brazil, between November, 1991, and December, 1993. Patient charts were reviewed to collect data on age, sex, region of the body affected, burn depth and surface affected, agent causing the burn, and circumstances in which the burn happened. Of the 138 cases, $71 \%$ occurred in the home. In $50 \%$ of all accidents the victims were children. Boiling water was the agent responsible for $59 \%$ of the accidents among children younger than 3 years of age; fuel alcohol used in the home for cooking and other purposes caused $57 \%$ of the accidents among children between 7 and 11 years, the most affected pediatric group. Among adults, the group between 20 and 39 years of age was the most affected (23\%); 84\% of the victims in this age group were male. Seventeen percent of all cases involved adult males at work. Women suffered burns in accidents at home $(67 \%)$ and in suicide-related events (33\%). Seventy-five percent of the suicide cases were women. The strategies to reduce the incidence of burn injuries should aim at eliminating environmental risk factors and implementing educational programs disseminated through radio and television. 